\title{
Multimedia Network Public Opinion Supervision Prediction Algorithm Based on Big Data
}

\author{
Yangfan Tong ${ }^{1}$ and Wei Sun (D) $^{2}$ \\ ${ }^{1}$ School of Art, Wuhan University, Wuhan 430072, China \\ ${ }^{2}$ School of Literature and Journalism, Hunan University of Technology and Business, Changsha 410205, China \\ Correspondence should be addressed to Wei Sun; 2748@hutb.edu.cn
}

Received 16 November 2020; Revised 9 December 2020; Accepted 14 December 2020; Published 22 December 2020

Academic Editor: Wei Wang

Copyright ( $) 2020$ Yangfan Tong and Wei Sun. This is an open access article distributed under the Creative Commons Attribution License, which permits unrestricted use, distribution, and reproduction in any medium, provided the original work is properly cited.

\begin{abstract}
This article focuses on the multidimensional construction of the multimedia network public opinion supervision mechanism, puts the research on the background of the era of big data, and based on the analysis and definition of the difference between network public opinion and network public opinion, deeply summarizes the network public opinion in the era of big data. New features analyze the opportunities and challenges faced by online public opinion in the era of big data. Based on the rational construction of the index system, this paper studies the multimedia network public opinion evaluation and prediction algorithm. Existing network public opinion assessment and prediction algorithms have shortcomings in capturing the characteristics of data sequences and the long-term dependence of data sequences, and the problems of overfitting and gradient disappearance may occur during training. Because of the above problems, based on the long-term and short-term memory network model, a regularized method is used to construct a multimedia network public opinion prediction model algorithm. This paper builds a multimedia network public opinion threat rating evaluation model based on the public opinion supervision prediction model and conducts analysis. The model constructed this time can not only improve the accuracy of public opinion assessment and prediction but also better avoid the problem of gradient disappearance and overfitting.
\end{abstract}

\section{Introduction}

Big datum is combined with all aspects of our lives, and the data explosion is a characteristic of the era we are currently facing. People participate and use the network, and they are generating data every moment. Managers in the new era must realize the value of data and pay attention to it. It finds effective methods to make data provide help and support for its own business [1]. The mingling of Internet public opinion and the Internet in the era of big data shows new features and brings greater management difficulty and challenges. At the same time, the continuous development and application of new technologies such as big data and cloud computing have brought support from data collection to analysis and processing into valuable information [2]. In the context of the new era, network public opinion can be regarded as a collection of opinion data of netizens, so it can also open up new situations through new technology revolutions [3]. While big data affects online public opinion, it also brings new opportunities and challenges to the building of network public opinion management capabilities [4, 5].

The combined development of big data and various fields not only brings new opportunities and challenges to various undertakings. At the same time, it also requires that network public opinion management requires innovative thinking and guidance. Big data, which specializes in data processing and refining information, has new room for discussion in connection with the management of online public opinion [6]. Many countries have begun to introduce big data in the management of online public opinion, but there are relatively few theoretical constructions that combine the two. In the past, the concepts and methods of network public opinion management faced shortcomings in the face of explosive data growth. It is no longer possible to use the 
thinking and methodology of passive emergency response after accumulating negative public opinion crises. Big data values relevance and connection and integrates big data thinking into the management of online public opinion. To sort out the existing research on the generation, the calm of Internet public opinion tries to combine it with big data concepts and technologies [7]. As shown in Figure 1, it discusses the application of big data in the extraction and classification of online public opinion, analysis, and monitoring, prediction and guidance, etc. Based on previous research on big data and network public opinion management theory, the depth of the combination of the two is further deepened and expanded.

Based on the research of network public opinion management under big data, it is not a simple technical proposal. Big datum is one of the key points of the future information society and academic research. It has strong vitality and development prospects [8]. It is both theoretical and practical at the technical level. Combining the tools with the research on online, public opinion can further improve the theoretical research on online public opinion, explore new forms of online public opinion in the new era, innovate the thinking and mechanism of online public opinion management, and improve the ability of online public opinion management [9]. Domestic research on the application of big data focuses on the use of big data technology for data mining, correlation analysis, and development of data value information. To get the application value of data, we study the proposition under the era of big data.

For the research on the application of big data in technology, it conducted a detailed review on the topics of big data acquisition, storage and management, transmission, processing, analysis and calculation, and application. This paper analyzes the current status of big data applications at home and abroad, highlights the current problems and deficiencies in China's big data technology and application research, and proposes targeted countermeasures [10]. For large numbers to explore the policy, according to technology and applied research in the development and improvement suggestions, we can have inspiration, and emphasize the strengthening of large data policy, technology, resource utilization, and sharing of these three areas to improve. In terms of big data and data association analysis, it has combed the current methods and paths of analyzing and mining data using big data technology and made a forecast of the application of big data technology in the direction of data association. It is emphasized that not only the technical level of big data applications should be concerned but also the long-term value and strategic role of data should be concerned [11]. This research should not only have reference significance for combining big data technology and mining methods with the field of network public opinion management but also enlighten the need to pay attention to the correlation analysis in big data.

Characteristics of big data association analysis are increasingly being tapped, and researches on the use of big data technology to build public opinion monitoring systems and prediction models to monitor and predict network public opinion have emerged. Based on the big data social

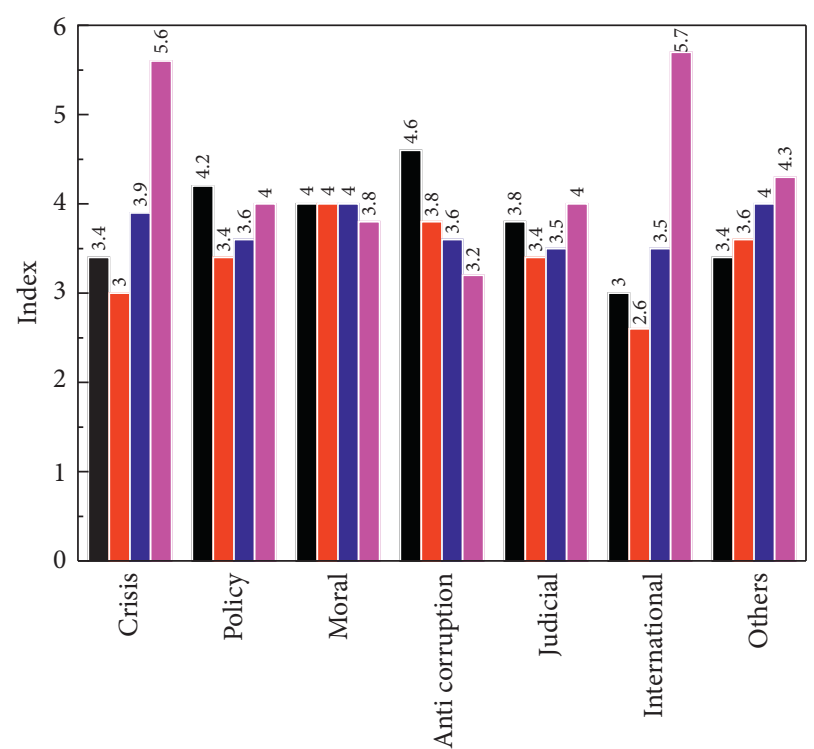

FIgURE 1: The consensus degree of various multimedia network public opinion events.

network analysis method for quantitative analysis of online public opinion, researchers focus on using big data to build a network public opinion analysis technology platform, emphasizing the pivotal role of the network "opinion leader," strengthening the cultivation of netizens' media literacy, and responding more actively and efficiently to the trend complex network public opinion spread. Researchers with similar characteristic analysis of large data and network data of public opinion focus on building a network for using the big data technology to forecast models. Related research analyzes the relevant concepts and characteristics of online public opinion under big data and builds a network public opinion analysis system model based on big data [12]. According to its research, the public opinion analysis system in a big data environment needs to satisfy the comprehensiveness of information capture, the timeliness of information processing, and the accuracy of analysis results. Based on these three requirements, a network public opinion analysis model includes four modules, which are information collection, information preprocessing, public opinion analysis, and public opinion report. In this process, big datadistributed acquisition technology, text preprocessing technology, and clustering technology are mainly used [13]. We discuss the application of big data technology to emergency public opinion information work, including public opinion monitoring and information collection, data processing of public opinion information, public opinion analysis, and event prediction support $[14,15]$.

This article uses the network public opinion assessment and prediction index system combined with a deep learning model to study the multimedia network public opinion assessment and prediction algorithm. Meanwhile, it introduces the relevant theories and technologies used by the multimedia network public opinion assessment and prediction research institute. The learning model is introduced in detail. Based on the data set of this paper, a suitable 
evaluation and prediction model are constructed, and finally, the model is applied to actual cases.

\section{Multimedia Network Public Opinion Supervision Subject and Process Based on Big Data}

2.1. Main Bodies of Network Public Opinion Management under Big Data. Under the big data, information dissemination media are innovative; various emerging media are emerging and widely used. The Internet media have become the main channel for the public to obtain information and voice, and the right to speak has changed. At the same time, the highly open network environment in the era of big data and the huge amount of data flowing at high-speed not only brings a large amount of original data of public opinion but also spawns new key nodes of public opinion. It reconstructs the existing discourse pattern and then influences the change of the network public opinion management subject. Big datum prompts frequent exchanges of information. The public accepts more foreign democratic ideals, the rule of law, and the concept of supervision. The public's civic awareness begins to awaken, and it begins to pay attention to various political topics related to its rights and interests. In the era of big data, all kinds of new media are convenient and fast to use, and the public can make speeches and publish information anytime, anywhere. You can also propose topics and initiate discussions. In the era of big data, new media have become an important public opinion field that cannot be ignored. With the help of new media "microphone" that everyone can use, netizens voluntarily publish new topics and discussions. These topics are often the focus of public attention nowadays, with a strong public opinion base and attractive discussion. The influence of public opinion generated by cyberspace is increasing.

As Internet politics continues to advance and become popular, direct dialogue between Internet users and the government is becoming more common. The influence of public opinion in cyberspace in government management decisions is getting older [16]. At some level, the ways and channels of the people's participation in political life have been changed. The highly open network environment under big data makes the public more actively participate in the discussion of public events and the deceleration of government management, which has a good effect on the progress and development of public space. But at the same time, it also poses great challenges to the management of online public opinion, for the high degree of openness of the network environment and the increasing expansion of the participating groups. The generation and development of online public opinion about an event are inevitably affected by countless different opinions. Because of the openness of the environment and the use of technology, the public has firmly grasped the right to speak in online public opinion, and its active participation has brought greater challenges and difficulties to the management of online public opinion.

In traditional paper media and the early stage of Internet development, the center of the mainstream media is to guide public opinion. At that time, public opinion occupies most critical management position in bonds, in addition to government administrative departments. Its grasp of the right to speak in dialogue is far higher than that of the public, and it serves as a bridge to create a good and healthy atmosphere of public opinion. As shown in Figure 2, with the rapid development of the Internet under big data, the mainstream media's voice is accompanied by the high participation of the people and the rise and strength of online media; the influence is weakened, and the participation of public opinion management has declined [17]. After discussing some emergencies or political topics, social media multiplies the topics with super spreading and spreading ability. Many popular topics are evenly spread over hundreds of millions of levels. However, the data generated in this process have shortcomings such as fragmentation, emotion, and one-sidedness. Therefore, the role of mainstream media is still not to be underestimated, and it needs to play its role as a store of public opinion. This shows that the extensive use of various new media has reduced the participation of traditional mainstream media in the management of online public opinion, but its role in public opinion management cannot be ignored.

With the arrival of the era of big data, all walks of life are increasingly paying attention to, attaching importance to, and applying big data technology and rationality to help carry out the work. A series of technological revolutions in the era of big data has also brought profound ideological updates [18]. The main body of development and utilization of big data should effectively establish the concept of big data and pay attention to the mining data value. The concept of "big data" is mainly to judge the development trend of things based on this concept with data. The judged beginning needs to be based on the overwhelming majority of valid data, analyzing ideas and methods to follow the real data analysis, mining the hidden value, summed up the law of development, and forecast the development trend of this process. In the past, in the management of online public opinion, the value of data was not as deeply understood as it is now, and the concept of the whole, macro, and refinement in big data was used to carry out network public opinion management.

With the application of big data in various fields, data value mining has enabled various businesses to develop better, and it has gradually been valued in the field of network public opinion management. The program collects and sorts out the keywords of netizens based on big data, analyzes the netizens' concerns, priorities, and demands and then uses big data visualization technology to talk about hot topics, which caused huge repercussions [19]. This shows that the use of big data can build a bridge between the public and administrative departments and not only let the public participate in public affairs management and decision-making but also build a good atmosphere of public opinion. The management department no longer regards big data as separate information technology but has big data thinking, attaches importance to data, mines data uses data, and allows data to help daily public opinion management. 



Figure 2: Distribution of social hotspot events in a multimedia network.

2.2. Features and Changes of the Network Public Opinion Management Process under Big Data. With the acceleration of the process of networking in modern society, the Internet has become an important place for the public to express personal opinions. Under the background of the big data environment, the mining and analysis of online public opinion information are more difficult than before and more difficult to standardize. The various technologies under big data can effectively dig out key factors from massive public opinion information and guide the response. The network public opinion management process generally includes finding public opinion, sorting and reporting, replying to the guidance, and tracking feedback. The network public opinion management process based on big data can optimize each link, and big data technology penetrates the data collection module, data preprocessing module, data clustering module, and information feedback module in the management process. Proper disposal and measures in place make the public opinion management process show the characteristics of timely discovery, rapid response, reasonable division of labor, and comprehensive feedback.

As shown in Figure 3, based on the big data network public opinion management process, it has changed the previous information collection to take questionnaires, interviews, and classified statistics as the basic data source. The public opinion analysis and judgment of the network obtain the generalized cognition by simple mathematical modeling, analysis function, and processing structure of single public opinion data. The online public intelligence sends a pattern in which the survey results are presented to decision-makers in the form of graphs. The process of network public opinion management based on big data focuses on collecting huge and diverse original public opinion data and then using data mining and artificial intelligence technology to obtain key information after cluster storage. Among them, big data has played an irreplaceable role.

Public opinion information collection is the first step of network public opinion management and is the process of obtaining public opinion information related data from the Internet. In the past, the collection of online public opinion information only focused on the simple and rough extraction of pages and data related to public opinion. It did not focus on the filtering of filtered, repetitive, and meaningless mixed data. Some data that did not have analytical value increased the online public opinion. The workload of analysis also interferes with the accuracy of the analysis. Based on big data-based network public opinion information collection, selecting appropriate web crawlers to crawl the corresponding web pages can effectively save the manpower and time of collecting information, after determining the source of information collection. It collects the filtered information as a data source for further analysis. The preprocessing of online public opinion information based on big data is divided into two processes. One is lexical item processing, including steps such as word segmentation, partof-speech tagging, and stop word filtering. The second is the key feature extraction, which can filter a large amount of redundant information and noise data in the data, extract keywords that can express the center, reduce the burden of public opinion analysis, and improve the analysis efficiency.

Because the current online public opinion information is often not a single piece of information but a collection of information on a series of related topics after preprocessing, cluster analysis in big data can be used to identify the central topic. This step includes quantifying the text data of the online public opinion and converting natural language into a form that the machine can understand. For different data types, you can choose the corresponding clustering algorithm to accurately classify different topics. It is convenient 


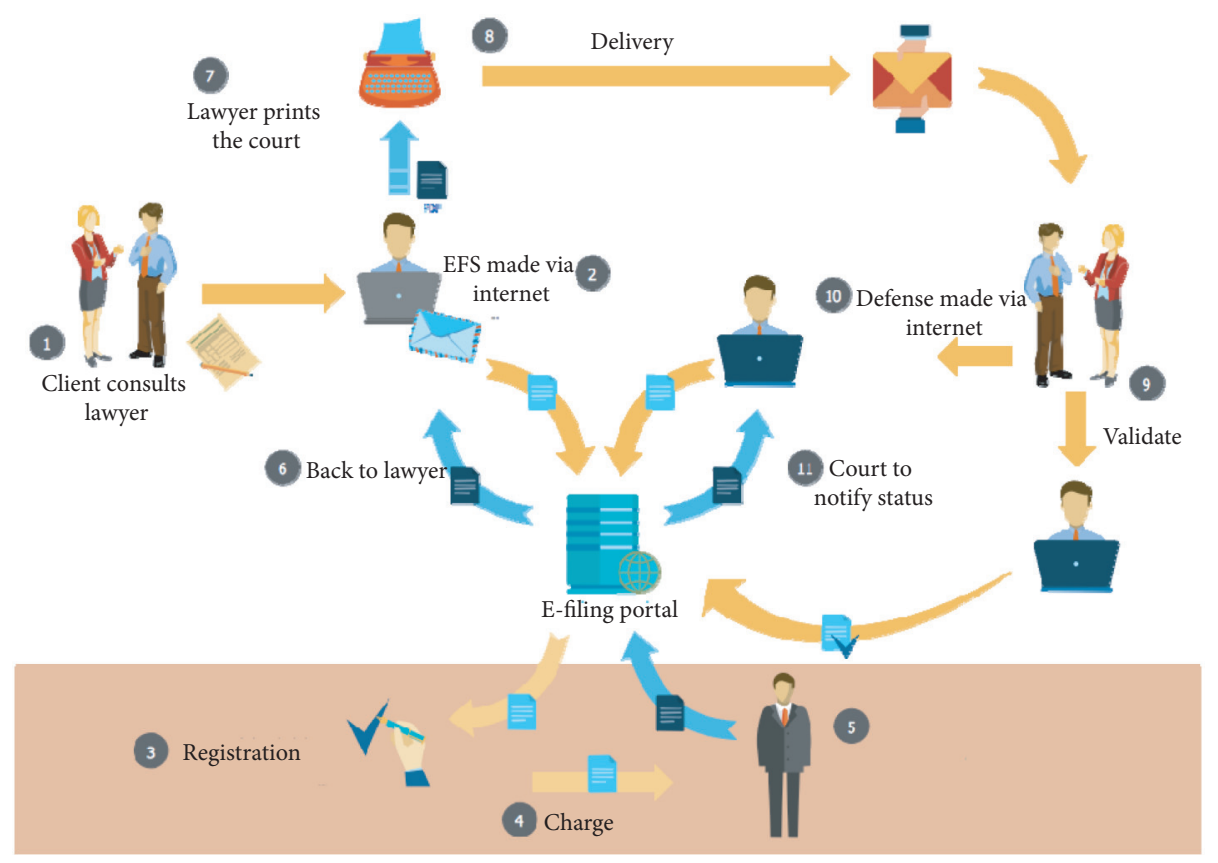

FIgURE 3: Multimedia network public opinion information processing flow.

to take corresponding measures for different topics. Finally, we can use the analysis technology of text sentiment intensity in big data to identify hot topics of public opinion and to make a timely prediction of public opinion. Using big data, you can build an online public opinion case database, match the hot topic of public opinion with the case database, and directly output the response plan when it is highly consistent. If it does not agree, the network public opinion under the big data analysis and sorting is not only threedimensional and comprehensive and presents clear, but also can sum up the public opinion management strategy for different situations to provide managers with reference choices. Every link of the network public opinion management process based on big data has been optimized, from the high-speed automatic collection of information, filtering and deduplication, clustering quantization to analysis and presentation, and output solutions, all showing unparalleled advantages.

In the past, network public opinion management mainly conducted a qualitative analysis of hot events, and public opinion analysts or managers interpreted from their own experience and theoretical knowledge accumulation [20]. As a result, the analysis results have a subjective color of personal experience and feelings, and even different institutions may draw opposite opinions on the same event. As shown in Figure 4, most of the original data can be used to develop new values through association analysis. The use of new values has transformed the management of online public opinion into quantitative management. Big data makes it possible to increase the value of data through scientific and rational mathematical calculations. It can interpret the data from multiple angles to take advantage of the hidden value of the data, so that the analyzed public opinion content is more comprehensive and accurate. Using big data technology can realize the intelligent analysis of network public opinion,

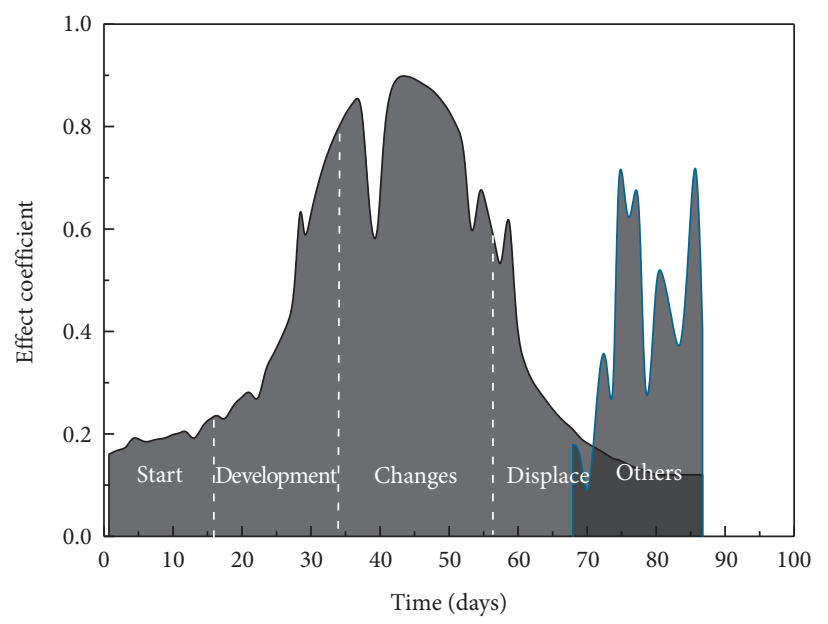

FIgURE 4: The development stage of multimedia network public opinion crisis.

and we automatically collect and capture data information related to the surface or deep layer of the target topic in 24 hours. After crawling and filtering, the prediction algorithm model automatically classifies the data, determines keywords, analyzes text, and researches and alarms. The sample data of research and judgment analysis based on big data can be expanded infinitely, which makes the judgment more accurate and the monitoring scope more extensive.

\section{Network Public Opinion Supervision Technology and Mechanism Based on Big Data}

3.1. The Application of Big Data Technology in the Supervision of Online Public Opinion. There is a correspondence 
between big data and the characteristics of online public opinion. Big data includes massive data and several data processing techniques and methods. Internet public opinion is not the data directly available on the Internet but the information obtained by mining and extracting the data. The collection and analysis of online public opinion coincide with big data. This shows that big data is consistent with the characteristics and goals of online public opinion. The core lies in a precise grasp of the present and accurate prediction of the future. This theoretically demonstrates the feasibility of combining them. With the exponential increase of network data, the traditional data processing technology has limitations, and it is helpless in the face of massive data. At the same time, it is stuck in the limitation of analysis methods and cannot predict the future development trend based on the current situation of network public opinion. Therefore, it is currently necessary to combine big data with network public opinion management to give full play to the professionalism of data technology [21].

Big data-based network public opinion management data is the foundation. Big data public opinion analysis is based on the analysis and mining of data, and because of the low data value density factor under big data, it can be obtained through analysis if enough data can be collected. The online public opinion is more comprehensive, accurate, and detailed. As shown in Figure 5, big data provides tools for massive data collection, which can use collection technologies such as web page information crawling of targeted sites, RSS summary collection, social networking site information collection, and full-text retrieval system. This refers to the use of a web crawler program to capture data from the online community with the most concentrated public opinion information and high discussion.

Aggregated content summary collection refers to the use of RSS summary information to collect data from news and blog websites. At the same time, it can aggregate many seeds that are closely related to the collection target and be able to know the dynamics of an event in an all-round way for the first time, mainly for real-time crawling and collection of media platforms that provide social network service systems. For example, the API uses a distributed Weibo crawler program to call the social network service API for information capture. It greatly facilitates the collection efficiency and breadth of information on netizens' social networks and provides a reliable data source for the next analysis of public opinion. The full-text retrieval system uses a full-text search engine to collect and retrieve multiple data types of a website via a protocol interface. The full-text retrieval system based on big data technology can use a specific protocol interface to establish a dynamic full-text retrieval intermediate library to meet the collection and retrieval needs of massive structured and unstructured data. When there is an upsurge of discussion on an event on the Internet, the powerful collection capabilities of the system are used to directly scan, search, and crawl the database and files of the website to achieve more effective online public opinion information collection.

Big data information processing technology is supported by HDFS storage, through the data warehouse tool HIVE and nonrelational distributed database HBase. By establishing a data index and keyword pool, a public opinion data warehouse is constructed and information data is loaded into the HBase database. The HBase replaces the traditional relational database with the nonrelational database NoSQL to solve the current large-scale and highly concurrent data processing problems of social networks. The data classification and integration technology contained in big data clusters and classifies network public opinion [22]. Cluster analysis is a process of dividing all data into multiple categories according to similarity. Through this process, data with high similarity can be aggregated and data sets with different types can have clear boundaries. There are currently two clustering methods. Process clustering can show changes in netizens' emotions, opinions, and attitudes. It grasped the evolution of public opinion to prevent a crisis of public opinion. Opinion clustering gathers similar viewpoints on the network and analyzes the attitude and opinion distribution and proportion of its subjects. Class analysis is to classify the data information after cluster analysis according to preestablished management indicators. As shown in Figure 6, the network public opinion supervision classifies the original information data after screening. It can be divided into categories such as environmental protection, justice, education, medical, and health and lays the foundation for the next step of network public opinion analysis.

Data mining technology is the key to big data. It refers to the development of price information that has not been discovered from massive, incomplete, fragmented, and random data. With the rapid development of network technology and computer technology, this technology can further deepen and use network data and optimize the management effect of network public opinion. Database, artificial intelligence, and statistical technology are important components of data mining technology, with functions such as correlation analysis, prediction, research judgment, and error analysis. It can improve the efficiency of data extraction and utilization, analyze the trend of public opinion contained in the data to the maximum extent, grasp the best time to deal with network public opinion, and greatly improve the processing speed of network public opinion. At the same time, it can also summarize the laws applicable to similar events from the previous data, and the specific model can accurately grasp the future public opinion evolution of such events. One of the methods of data mining that is closely combined with online public opinion is the opinion mining method. This method is mainly to extract the subject and opinion of the text ontology constructed in the mining field. By constructing a polar dictionary, the emotional tendency of keywords is analyzed, and the emotional tendency of the target text is finally obtained by combining various methods. Under the background of big data technology, the acquisition of emotional vocabulary and the quantitative analysis of polarity are changed from manual judgment to the use of corpus to calculate the similarity of vocabulary tendencies. You can also use linguistic knowledge to quantitatively analyze the constituent characteristics of words. 


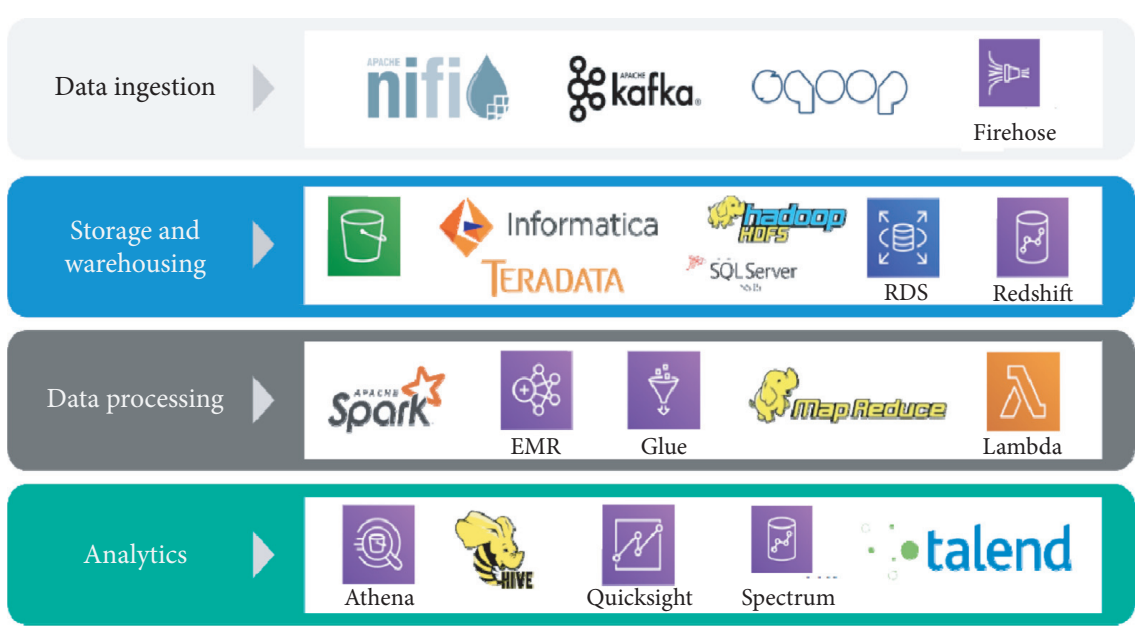

Figure 5: Big data technology classification.

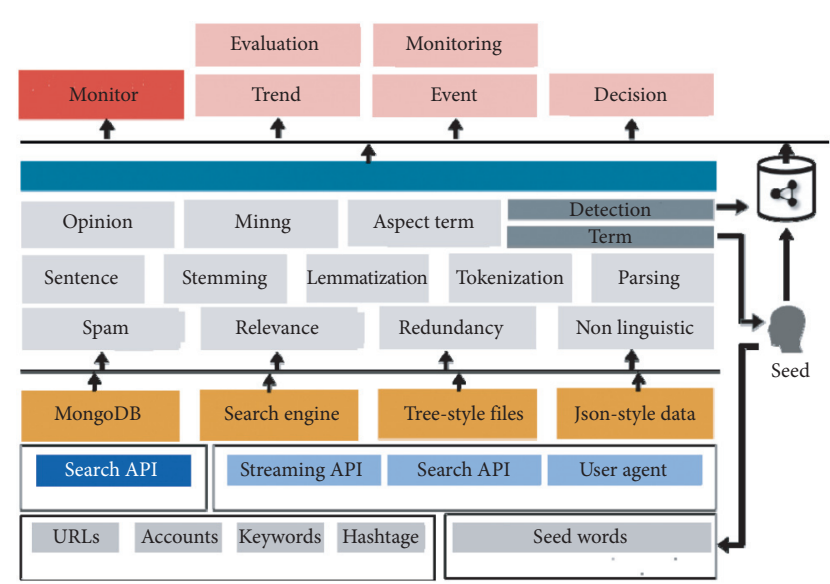

Figure 6: Multimedia network public opinion data collection structure diagram.

Text sentiment techniques include sentiment analysis and text sentiment analysis. Emotional tendency analysis can deepen the semantic understanding of netizens' speech to determine their emotional attitude to the entire topic. It helps analyze the current sentiment of netizens, the popularity of topics, and the trend of events. Using opinion mining methods, the collective emotions implied in public opinion data can be analyzed to predict the evolutionary trend of emotions.

3.2. Multimedia Network Public Opinion Supervision Mechanism under Big Data. Under the big data of the network public opinion ecological environment, the public opinion management structure needs to break through the traditional model of the government singing "one-man play" and explore the related structure of innovative social subjects. The main coping mechanisms of public opinion played an effective way to build a network of public opinion management. The basis of big data network public opinion management is the sharing and circulation of data resources. However, for a long time, there have been big data interconnection breakpoints on the block boundaries of social organizations and government agencies in China. The information-sharing of the same event is indifferent public opinion management departments divided by blocks. In the actual operation process, the central and local governments, research institutions, and media have set up corresponding institutions for monitoring and managing online public opinion. The division of organizations and their levels are clear, which leads to the cross-operation of supervisory responsibilities and the linkage of network public opinion management. The "pyramid"-type monitoring system and vertical management system have a strong exclusive tendency; the value function of external agents is ignored; the interaction and collaboration between management agents are not enough, and the response is weak. The management structure of vertical management and step-by-step reporting has resulted in poor communication between management departments, resulting in the phenomenon that the data information barriers are blocked and sharing is insufficient. It placed the number of management levels and different sectors of regulation databases in the type of data storage, semantic expressions, and other hardware support apparatus that exist. Thus, the database system to the various organs is difficult to effective convergence. Driven by the interests of departments and the lack of awareness of sharing, when the crisis of online public opinion emerges, the network public opinion response mechanism cannot achieve the integration of all data information, and the efficiency of resource utilization is low.

On this basis, the network public opinion response mechanism under big data should be reformed and innovated, requiring the management department to adopt a flattened and grid-like organizational structure model, so that various public opinion institutions and participating entities can collaborate in the process of network public opinion management interactive. The large-scale network public opinion vertical management structure model can save the management costs, expand the scope of public opinion data resource acquisition and management regulation, and improve the management effectiveness. This 
management structure combines the life cycle division of network public opinion. The first stage is to track and monitor the network public opinion in the budding period in real time. The second stage is the signal screening of the online public opinion that has entered the public's field of vision and started to rapidly ferment. In the third stage, the audience, content, and influence of the degree of public opinion should be aggregated and analyzed to judge the development trend of public opinion. Once the negative public opinion is turned to reach the minimum value of risk prediction, the fourth stage of damage control and timely grooming mechanism is adopted to push public opinion into a period of decay and calm. The fifth stage retrospects and summarizes the disposition of public opinion after quelling and establishes a network public opinion file for reference.

The public opinion data under big data is highly complicated, and the development situation is uncertain and uncontrollable, which promotes the network public opinion decision-making mechanism to gradually change the negatively controlled network public opinion management method in the past. Big data is involved in the network public opinion decision-making mechanism. From the perspective of the data nature of online public opinion decision-making, big data deconstructs and reconstructs the fragmented and unsystematic massive public opinion data collected, fully integrates and deeply integrates the network public opinion data resources, and breaks up the surface form of mixed data. With the help of big data visualization technology, it can be displayed to the management department understandably and more intuitively for serving public opinion decision-making. The use of big data can improve the analysis and information service capabilities of online public opinion data, absorb reasonable and most public opinion into the network public opinion management decision-making mechanism, and realize the legitimacy of it. At the same time, big data can provide management departments with a large amount of real-time and accurate decision information through the analysis of hot spots and focus issues of public attention, thereby improving the management department's ability to predict the occurrence and degree of network public opinion events, trends, and results. The network public opinion decision-making mechanism can plan the decision-making plan, greatly improving the decision-making efficiency. The analysis of online public opinion big data promotes the change of online public opinion decisionmaking from qualitative to quantitative and promotes the decision-making to rely more and more on rational judgment and data analysis rather than experience or even intuition.

\section{Multimedia Network Public Opinion Supervision Prediction Algorithm Model Construction and Analysis Based on Big Data}

4.1. Construction of Multimedia Network Public Opinion Assessment and Prediction Model. To accurately research multimedia network public opinion evaluation, it is very important to construct a scientific multimedia network public opinion index system and build an excellent evaluation model based on the index system. This article introduces the index system that has given the quantitative methods of each index. And, multimedia network public opinion predicts different multimedia network public opinion on the threat level assessment. It is already happening multimedia events threaten extent of discrimination that happened multimedia event threat level for accurate positioning. As shown in Figure 7, this paper builds a multimedia network public opinion threat rating model based on the modified BiLSTM of the LSTM long-shortterm memory network. The BiLSTM model is a combination of forwarding LSTM and backward LSTM, which can better capture the internal relationship between time series features before and after public opinion data. Compared with CNN, BiLSTM can better capture the context of multimedia data. Compared with the RNN model, the BiLSTM model can better avoid gradient disappearance and gradient explosion problems. Compared with the LSTM model, BiLSTM can make the training faster and achieve a fuller learning effect.

Using python crawler technology to crawl multimedia network public opinion data from weibo, the data set mainly contains fields such as title, content, retweets, likes, and comments. The title and content belong to the text data. The word segmentation tool is used for Chinese word segmentation first, and the word segmentation is expressed by the Skip-Gram model for word vector representation. This article uses the final index of the index system as the input of the model. The final index refers to the last subindex of various indicators. Among the type of topic and the influence of the publisher, the degree of sensitivity of public opinion information content is a qualitative indicator, whether there are rumors. The subject content belongs to long text data and is expressed using word vectors. After quantizing each of the last-level indicators, a weibo vector or news vector is formed and trained using the BiLSTM model. The BiLSTM model is a supervised classification model. The model is trained using labeled data to obtain appropriate model parameters, and then the trained model is used on the test data set. This article's multimedia network public opinion evaluation results in the general threat level, heavy and serious, particularly serious.

As shown in Figure 8, the results of the BiLSTM model are superior to those of other models. In the case where the same data set and the number of iterations are equal, the BiLSTM model can capture the characteristics of the multimedia network public opinion data sequence and the longterm dependence of the data sequence compared with the separate $\mathrm{CNN}$ and RNN models. This paper uses dropout regularization. Combined with LSTM compared to the model, they can better avoid overfitting and gradient problems disappear so that the multimedia network public opinion threat level assessment is more accurate. Under the platform multimedia data set, the paper uses the final indicators of the index system as the input of the BiLSTM model and only uses the fields of the title, content, source, number of reposts, likes, and comments in the data set as the 


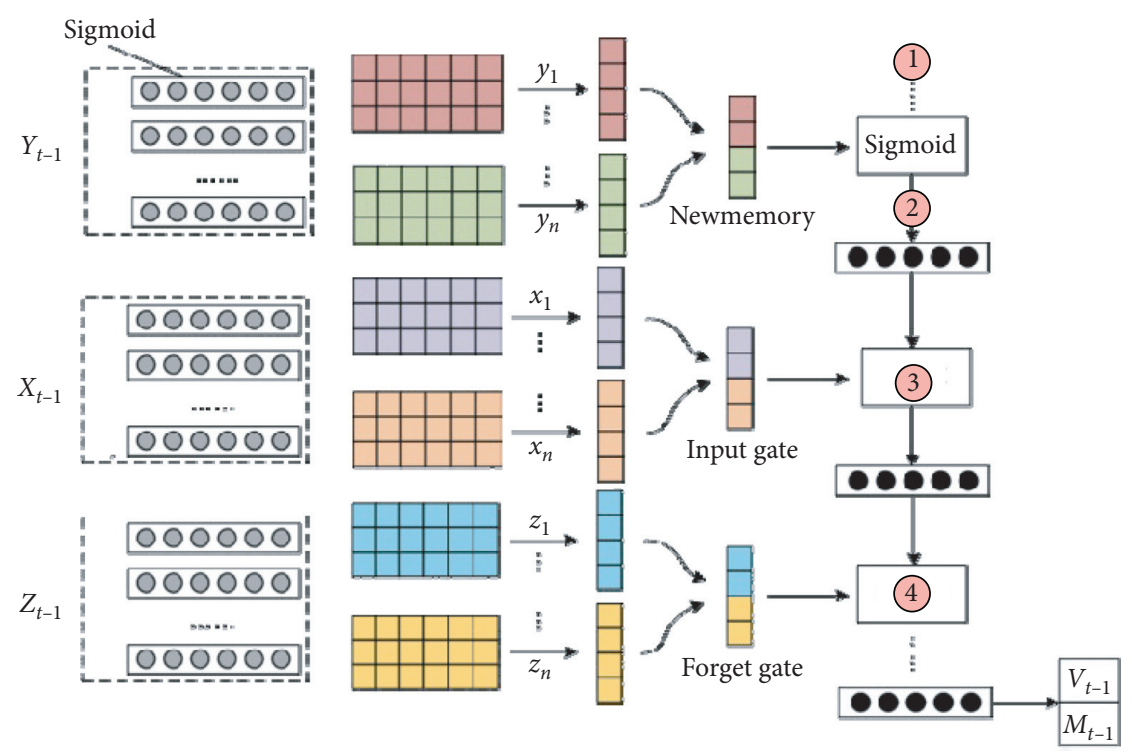

FIGURE 7: Schematic diagram of the LSTM model structure, and the symbols of $x, y, z, X, Y, Z, V$, and $M$ are the calculation parameters of the model.

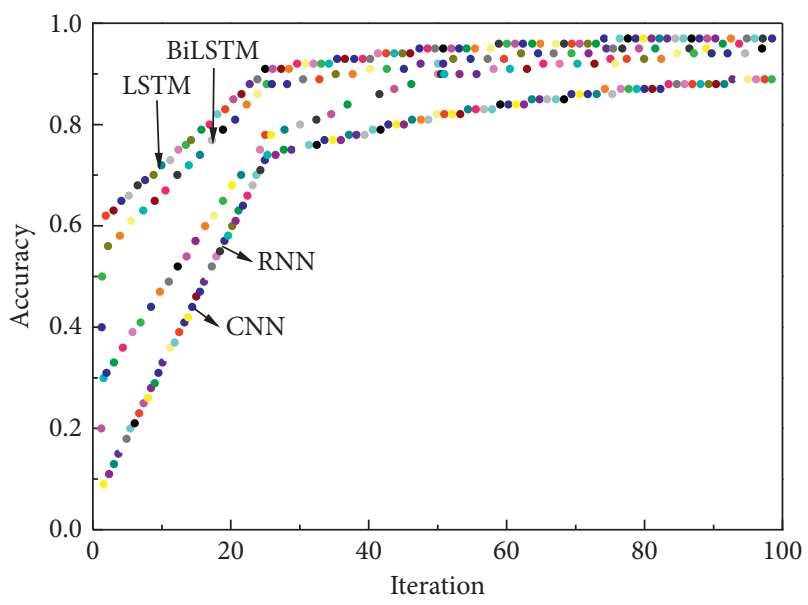

FIGURE 8: Model iteration times and accuracy curve.

input of the BiLSTM model for comparison. In this study, we conduct a multimedia network public opinion threat assessment study and first introduce network public opinion data collection, followed by a detailed description of data preprocessing methods. Finally, the BiLSTM model is compared with the CNN, RNN, and LSTM models. The model in this paper has the advantages of CNN, RNN, and LSTM models at the same time. The results also show that the BiLSTM model has a better evaluation effect on the threat level of the multimedia network public opinion.

4.2. Multimedia Network Public Opinion Supervision Prediction Algorithm Evaluation and Public Opinion Analysis. Under the collected data set in this article, the final index of the indicator system in this article is used as the input of the Re-LSTM model. The solid line is the prediction accuracy curve using the index system. In the case of the same data set and the same number of iterations, the ReLSTM model input can more fully grasp the sequence characteristics of public opinion data, and the prediction result of network public opinion is more accurate. The optimization in both places can improve the convergence effect and speed of the LSTM network to a certain extent. Experimental results show that the Re-LSTM model in this paper not only improves the accuracy of public opinion prediction for multimedia networks based on big data but also improves the convergence effect and speed of LSTM networks.

To more intuitively see the evaluation results and prediction results of the multimedia network based on big data obtained by the algorithm model analysis, the results need to be displayed in the form of web page charts through the web layer, so this paper developed a multimedia network public opinion evaluation and prediction system based on big data. The system consists of center users, the negative analysis, the overall trend of public opinion, and evaluation and forecasting the results of public opinion and reporting modules, of which this paper shows based on a large multimedia data to assess public opinion and network prediction after analyzing the results of the core modules. This system mainly uses Echarts combined with the element component library to display the evaluation and prediction results. The picture is the result of the negative analysis module. This module is mainly to show the proportion of neutral, mildly negative, moderately negative, and severely negative public opinion information over a while. This is an assessment of the current state of network public opinion and future development. It shows the general development trend of public opinion and reflects the current situation of public opinion events in multiple media. As shown in Figure 9, you can intuitively see the current state of public opinion events and speculate future trends. 


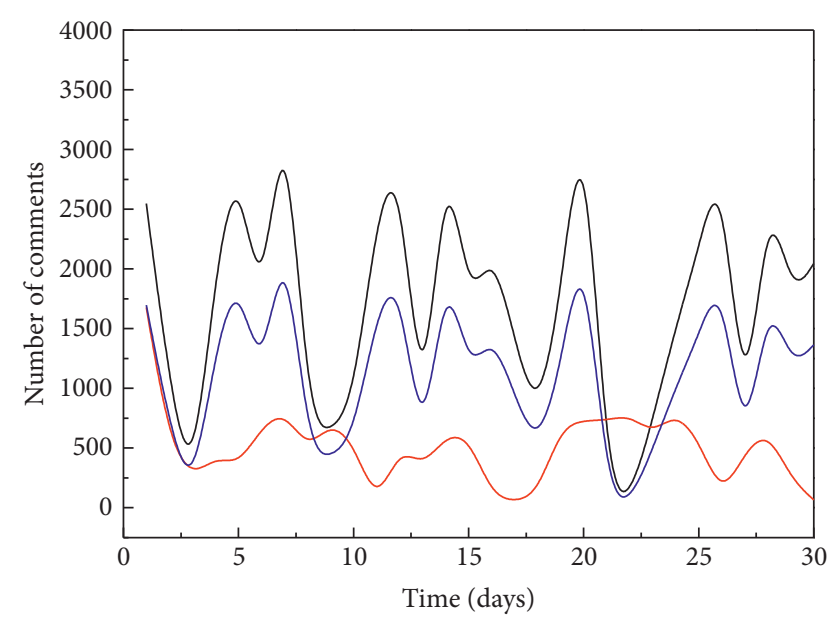

FIGURE 9: Distribution of the general trend of multimedia network public opinion.

\section{Conclusion}

Multimedia network public opinion evaluation is based on big data. A reasonable and measurable index system is the basis and primary condition for building a multimedia network public opinion assessment and prediction model based on big data. The index system makes up for the shortcomings of the previous index system regarding public opinion themes and evolutionary communication.

Using the index system and quantization method for data preprocessing, and then based on this index system and BiLSTM algorithm, this paper aims to build BiLSTM big data-based multimedia network public opinion threat rating model. The BiLSTM evaluation model is better than the fuzzy mathematical evaluation model and the traditional BP neural network model. The BiLSTM threat level evaluation model constructed in this paper has a strong feature extraction capability and can be better learned based on a large scale than the LSTM model. The long-term dependence of public opinion data in the multimedia network of data can better avoid the problem of overfitting, after the regularization of dropout during the training process. Based on the preprocessed data, the Re-LSTM prediction model is constructed, and finally, a system is developed to display the evaluation and prediction results.

Although this article proposes a complete set of big databased multimedia network public opinion assessment and forecasting index system, there are still some incomplete considerations. For the qualitative indicators, this article uses a questionnaire to quantify; there is a small amount of chance, which may have a certain impact on the quantitative results. In multimedia large data based on the network, public opinion evaluation and prediction build a model aspect as used herein regularization, as the method penalty LSTM input weight of the objective function has been improved. We constructed Re-LSTM model applied on the data multimedia network public opinion prediction problem which has also achieved ideal results. According to the analytical result curve, we can know that, with the increase of the number of iterations, the experimental accuracy rate is increasing but the growth rate is very small. Whether there is a better model improvement strategy that can make the experimental curve change more obvious is the research work of the subsequent topic.

\section{Data Availability}

The data used to support the findings of this study are available from the corresponding author upon request.

\section{Conflicts of Interest}

The authors declare that they have no conflicts of interest regarding the publication of this paper.

\section{References}

[1] H. Zou, a. Li, C. Ao, P. Zhang, N. Li, and Z. Wang, "Task classification-aware data aggregation scheduling algorithm in wireless sensor networks," International Journal of Mobile Network Design and Innovation, vol. 9, no. 2, pp. 106-117, 2019.

[2] Y. Liu, Q. Li, X. Tang, N. Ma, and R. Tian, "Superedge prediction: what opinions will be mined based on an opinion supernetwork model?" Decision Support Systems, vol. 64, pp. 118-129, 2014.

[3] A. J. Langdon, M. J. Sharpe, G. Schoenbaum, and Y. Niv, "Model-based predictions for dopamine," Current Opinion in Neurobiology, vol. 497 pages, 2018.

[4] D. Z. Wei, F. J. Chen, and X. X. Zheng, "Internet public opinion chaotic prediction based on chaos theory and the improved radial basis function in neural networks," Acta Physica Sinica, vol. 64, no. 11, pp. 110-123, 2015.

[5] T. H. Nguyen, K. Shirai, and J. Velcin, "Sentiment analysis on social media for stock movement prediction," Expert Systems with Applications, vol. 42, no. 24, pp. 9603-9611, 2015.

[6] M.-Y. Cheng and A. F. V. Roy, "Evolutionary fuzzy decision model for cash flow prediction using time-dependent support vector machines," International Journal of Project Management, vol. 29, no. 1, pp. 56-65, 2011.

[7] H. Valipour, F. Salehi, F. Salehi, and M. Bahrami, "Predicting audit reports using meta-heuristic algorithms," Journal of Distribution Science, vol. 11, no. 6, pp. 13-19, 2013.

[8] D. Bokde, S. Girase, and D. Mukhopadhyay, "Matrix factorization model in collaborative filtering algorithms: a survey," Procedia Computer Science, vol. 49, no. 2, pp. 136-146, 2015.

[9] D. Acemoğlu, G. Como, F. Fagnani et al., "Opinion fluctuations and disagreement in social networks," Mathematics of Operations Research, vol. 38, no. 1, pp. 1-27, 2013.

[10] J. Zhou, X. Li, and X. Shi, "Long-term prediction model of rockburst in underground openings using heuristic algorithms and support vector machines," Safety Science, vol. 50, no. 4, pp. 629-644, 2012.

[11] C. Catal and M. Nangir, "A sentiment classification model based on multiple classifiers," Applied Soft Computing, vol. 50, no. 1, pp. 135-141, 2017.

[12] M. A. Saad, A. C. Bovik, and C. Charrier, "Blind prediction of natural video quality," IEEE Transactions on Image Processing, vol. 23, no. 3, pp. 1352-1365, 2014.

[13] H. Liu, J. He, T. Wang, W. Song, and X. Du, "Combining user preferences and user opinions for accurate recommendation," 
Electronic Commerce Research and Applications, vol. 12, no. 1, pp. 14-23, 2013.

[14] B. B. Doll, D. A. Simon, and N. D. Daw, "The ubiquity of model-based reinforcement learning," Current Opinion in Neurobiology, vol. 22, no. 6, pp. 1075-1081, 2012.

[15] J. Patel, S. Shah, P. Thakkar, and K. Kotecha, "Predicting stock and stock price index movement using trend deterministic data preparation and machine learning techniques," Expert Systems with Applications, vol. 42, no. 1, pp. 259-268, 2015.

[16] Y. Wu, S. Liu, K. Yan, M. Liu, and F. Wu, "Opinion flow: visual analysis of opinion diffusion on social media," IEEE Transactions on Visualization and Computer Graphics, vol. 20, no. 12, pp. 1763-1772, 2014.

[17] H. Chiroma, S. Abdulkareem, and T. Herawan, "Evolutionary neural network model for West Texas Intermediate crude oil price prediction," Applied Energy, vol. 142, pp. 266-273, 2015.

[18] C. Laing and T. Schlick, "Computational approaches to RNA structure prediction, analysis, and design," Current Opinion in Structural Biology, vol. 21, no. 3, pp. 306-318, 2011.

[19] H. Oh, S. Lee, and A. C. Bovik, "Stereoscopic 3D visual discomfort prediction: a dynamic accommodation and vergence interaction model," IEEE Transactions on Image Processing, vol. 25, no. 2, pp. 615-629, 2015.

[20] W. Wang, M. Carr, W. Xu, and K. Kobbacy, "A model for residual life prediction based on Brownian motion with an adaptive drift," Microelectronics Reliability, vol. 51, no. 2, pp. 285-293, 2011.

[21] N. Gordini, "A genetic algorithm approach for SMEs bankruptcy prediction: empirical evidence from Italy," Expert Systems with Applications, vol. 41, no. 14, pp. 6433-6445, 2014.

[22] G. Baio and M. Blangiardo, "Bayesian hierarchical model for the prediction of football results," Journal of Applied Statistics, vol. 37, no. 2, pp. 253-264, 2010. 